\title{
Odor-mediated alleyway performance as a function of squad position with varied donors
}

\author{
RONALD D. TAYLOR \\ Salem College, Salem, West Virginia \\ and \\ H. WAYNE LUDVIGSON \\ Texas Christian University, Fort Worth, Texas
}

\begin{abstract}
Squads of three rats were administered rewarded and nonrewarded trials in a straight alleyway, with all three members getting the same type of goal event on a given trial. Both the membership of the squads and the position of individual rats within the squads were randomized across days. Run speeds were examined as a function of the type of goal event and the squad position. Speeds on rewarded trials did not change as a function of squad position. Speeds on nonrewarded trials decreased increasingly when rats occupied the second and third squad positions. The tendency to run relatively slowly in the third position on nonrewarded trials was inversely related to the amount of training rats had received in the first squad position. The results support the inference that odor emissions from rats on nonrewarded trials are similar from rat to rat, rather than idiosyncratic, that they accumulate, and that greater concentrations provide more effective discriminative stimuli.
\end{abstract}

Numerous studies have supported the hypothesis that laboratory rats excrete odors in response to rewarded and nonrewarded treatments (e.g., Ludvigson, 1969; McHose \& Ludvigson, 1966; Mellgren, Fouts, \& Martin, 1973; Morrison \& Ludvigson, 1970; Prytula \& Davis, 1974; Taylor \& Ludvigson, 1980a, 1980b). One procedure employed to study this phenomenon involves patterned responding in a straight alleyway (Ludvigson \& Sytsma, 1967). With this procedure, a series of rewarded and nonrewarded trials are administered to a squad of rats, with each rat receiving the same goal event on a given trial, and the rats are tested in rotation such that all rats receive the first trial before any rat receives the second, and so on. Utilizing a schedule of rewarded and nonrewarded goal events that exceeds the memory capacities of the rat (Capaldi, 1971) and removing odors only after the entire squad has completed a trial, a pattern of fast running on rewarded trials and slow running on nonrewarded trials emerges over trials. This patterned responding is largely due to differential conditioning controlled by discriminatively different odors emitted on rewarded (" $R$ odors") and

This research was made possible in part by a fellowship awarded to the first author by the Texas Christian University Research Foundation and by a research grant awarded to the second author. Requests for reprints should be sent to Ronald D. Taylor, who is now at the Department of Psychology, University of Kentucky Center at Fort Knox, Fort Knox, Kentucky 40121. nonrewarded ("N odors") trials (Eslinger \& Ludvigson, 1980a).

One issue of some importance concerns the extent to which the emitted odors are the same from rat to rat and therefore accumulate as successive rats experience one of these goal events. Eslinger and Ludvigson (1980b) found that changing the membership of an odor-donor squad did not appreciably alter patterned responding in test rats that followed them in the alleyway, and suggested that the odors possess common characteristics across donors. Seago, Ludvigson, and Remley (1970) found that rats permanently assigned to the later positions in a squad of eight rats exhibited greater differences in goal speeds between rewarded and nonrewarded trials than rats assigned to earlier positions. More recently, Prytula, Davis, and Fanning (1981) demonstrated that rats regularly preceded by nine or more conspecifics ran faster on rewarded trials and more slowly on nonrewarded trials than did other rats preceded by no more than four conspecifics.

Although both the Prytula et al. (1981) and the Seago et al. (1970) studies are consistent with the inference of an accumulation of a common odor across rats, other inferences are possible. For example, the emission could be idiosyncratic and of different salience, with rats in later squad positions realizing an advantage because of the greater likelihood of at least one emission being highly salient and, thus, readily associated with the goal event. 
Alternatively, the greater number of distinctive odors available to rats in later squad positions might provide a better stimulus, either as an aggregrate or through perceptual synthesis. Such use of idiosyncratic emissions should be difficult, however, to the extent that different emissions are present from trial to trial, because the rat must learn the significance of them.

In the Prytula et al. and Seago et al. studies, squad membership and a rat's position in the squad were constant, thus providing a stability of emissions over trials. In the present study, they were varied so as to permit an examination of patterned responding for each rat as a function of squad position under conditions in which discriminative odor emissions come from rats selected randomly from a pool from session to session.

\section{METHOD}

\section{Subjects}

The subjects were 12 male albino rats obtained from the Holtzman Company at 99 days of age. Upon arrival, they were individually housed in wire-mesh cages and provided free access to food and water. At 131 days of age, they were placed on a restricted feeding regimen and gradually reduced in body weight until stabilized between $80 \%$ and $85 \%$ of their free-feeding weights. They were maintained at this level throughout the experiment by being fed an appropriate amount of food at the end of each experimental session.

\section{Apparatus}

A single straight alleyway, constructed of stainless steel and covered by a glass top, served as the apparatus. The alleyway was $7.62 \mathrm{~cm}$ wide and $20.3 \mathrm{~cm}$ high, and consisted of a $25.4-\mathrm{cm}-\mathrm{long}$ start section and a $46.6-\mathrm{cm}-$ long run section separated by a sliding stainless steel door. Measurement of the rat's latency of traversal was accomplished by using microswitches connected to the startdoor and a floor panel located $10 \mathrm{~cm}$ from the goal end of the alleyway. Opening of the startdoor started a timer that was stopped when the weight of the rat depressed the floor panel, yielding a single latency through $36.6 \mathrm{~cm}$ of the alleyway.

Covering the floor of the alleyway was adding-machine paper, which was fed by a roll affixed outside the startbox end, came up through a slit in the floor of the startbox, and went out through a slit at the goalbox end. On all trials, a removable glass food cup was kept on the paper flooring between the floor panel and the end of the alleyway. The cup was positioned so that $45-\mathrm{mg}$ Noyes pellets could be dispensed into it through a glass spout that protruded through the end of the alleyway.

The alleyway was cleaned by activating a vacuum cleaner, attached at the goalbox end, and drawing room air in through an opening covered by wire mesh in the startbox end. The air exhausted from the alleyway was expelled to the outside of the building via a nearby window. Additional cleaning involved pulling the paper covering the floor through the slit in the goalbox end until the entire alleyway was covered with fresh paper.

\section{Procedure}

Following preliminary handling and adaptation to the alleyway, training began. The 12 rats were divided randomly into four squads of 3 each. Two of these squads were then selected randomly to receive alleyway training on the 1 st day, leaving the remaining two squads to receive training on the following day. On the 3rd day, four new squads were formed from the original pool of 12 rats. This procedure of randomly forming four squads on alternate days and running two of the four squads each day continued throughout the experiment. Thus, each rat received training on 2 days of every 4 and was never idle for more than 2 consecutive days. The random assignment of rats to squads served to randomize both membership in a squad and the position of a given rat within a squad across days. Within a day of training, squad membership and squad position remained constant.

To administer a trial to a squad of rats, the first member of the squad was carried into the room containing the alleyway, removed from its home cage, and placed into the startbox facing away from the startbox door. When the rat turned and faced the door, the door was lifted, allowing the rat to traverse the alleyway. On rewarded trials, 15 pellets were automatically dispensed when the rat stepped on the floor panel located just in front of the food cup, a stopwatch was started, and the rat was allowed $30 \mathrm{sec}$ to consume the pellets. On nonrewarded trials, no pellets were dispensed, and the rat was confined to the goalbox for $30 \mathrm{sec}$. After being removed from the goalbox, the rat was removed to another room before the next rat was carried into the experimental room.

Each training day, all rats were given eight trials-four rewarded (R) and four nonrewarded (N). The order of $R$ and $N$ trials was determined randomly each day, with the restriction that not more than three $\mathbf{R}$ or three $\mathbf{N}$ trials occur in succession. The first trial of the day was administered to all three members of a squad before any member received the second trial, and so forth; however, the three members of a given squad received all eight of their trials before the second squad of the day was administered any trials. The alleyway was cleaned between squad trials and squads, but not between animals within a trial.

\section{RESULTS}

Reciprocals of the latencies for each rat on each trial were multiplied by the appropriate constant to yield speeds in meters per second. Mean $\mathbf{R}$ and $\mathbf{N}$ speeds for each rat within a session were computed, and the means of such means for rats run in the second and third squad positions are depicted in Figure 1 . These data were not analyzed statistically, because the particular rats given training varied from day to day. However, inspection of the figure indicates that, consistent with previous studies, differential runs speeds emerged after some amount of training.

Beginning with Session 18, the mean $R$ and $N$ speeds for individual rats across the remaining days

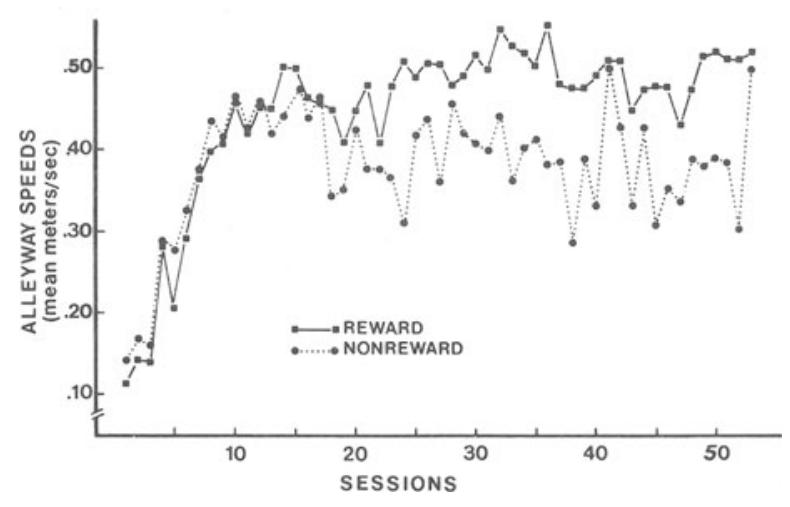

Figure 1. Mean alleyway speeds on rewarded and nonrewarded trials for rats occupying the second and third squad positions. 
of the experiment were computed separately for each of the three squad positions. These (unweighted) means were then used as units in the following analysis, because individual rats differed somewhat in the number of times they had occupied a particular squad position. Means of these units, along with standard deviations, are depicted in Figure 2. A repeated-measures analysis of variance on these data, with goal event ( $R$ vs. $N$ ) and squad position $(1,2,3)$ as repeated measures, produced significant effects for goal event $[F(1,55)=15.72]$, squad position $[F(2,55)=$ 6.55 , and goal event $\times$ squad position interaction $[F(2,55)=5.24]$; all $p$ values $<.01$. Follow-up analyses indicated that the $\mathbf{R}$ vs. $\mathbf{N}$ difference was not significant at the first squad position, but was significant at the second $[\mathrm{F}(1,55)=6.63, \mathrm{p}<.05]$ and third $[F(1,55)=19.02, p<.05]$ squad positions. In addition, run speeds on $R$ trials did not change significantly as a function of squad position, although speeds on $\mathbf{N}$ trials did decrease as a function of squad position $[F(2,55)=11.65, p<.05]$.

Alleyway speeds for each of the 12 rats in the pool were plotted as a function of squad position and goal event and are presented in Figure 3. Inspection of of these data indicates that faster run speeds occurred on $R$ trials than on $\mathrm{N}$ trials, in 4,10 , and 12 rats as a function of their occupying the first, second, and third squad positions, respectively. Furthermore, the magnitude of the difference between $\mathbf{R}$ and $\mathbf{N}$ trials was greater for the third than for the second squad position for 11 of 12 rats, with the two differences being equal for the 12th rat. This result is significant at the .001 level by the sign test. Rather large individual differences are also obvious, suggesting substantial differences among rats in the ability of $\mathbf{R}$ and $\mathrm{N}$ odors to control differential responding.

To obtain information on the relationship between

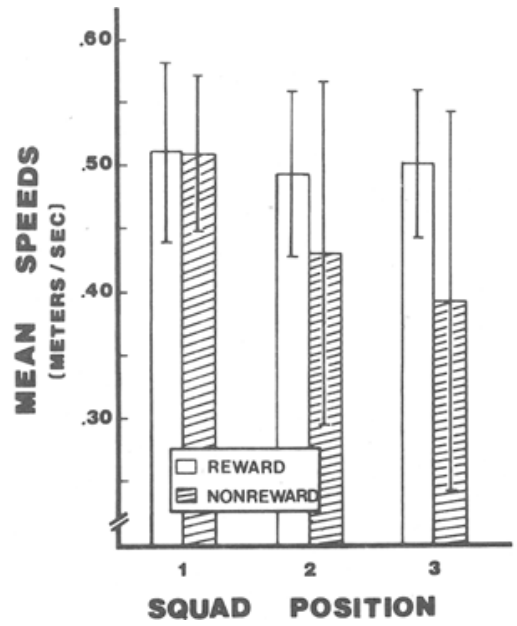

Figure 2. Means and standard deviations for alleyway speeds on rewarded and nonrewarded trials, computed across all rats, as a function of squad position during Sessions 18 through 53.

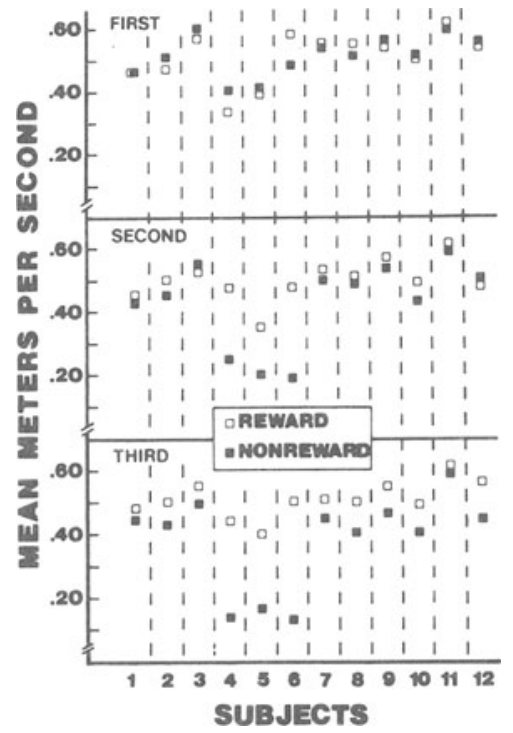

Figure 3. Mean alleyway speeds on rewarded and nonrewarded trials for each rat, computed over Sessions 18 through 53, as a function of occupying the first (upper panel), second (middle parel), and third (lower panel) squad positions.

these individual differences and the discrimination training procedure, the mean differences between $\mathbf{R}$ and $\mathbf{N}$ speeds $(\mathbf{R}-\mathbf{N})$ for individual rats when run in the third squad position were correlated with the number of training sessions in which the rats held the first squad position. This analysis produced a significant Pearson $r$ of $-0.84, p<.01$. This large negative correlation suggests that the more trials a rat had without benefit of odor cues, that is, occupying the first squad position (or the fewer trials with benefit of odor cues, since these covaried), the less was the discriminative control exercised by the odor cues when the rat held the third squad position.

\section{DISCUSSION}

Perhaps the most significant finding of this study is the mere appearance of response patterning when rats occupied the second and third squad positions: significance of the odors was learned in spite of the fact that a third of the trials, on average, had no differential odor cues available, and in spite of the fact that the odors were, from day to day, from different rats, each having its own "signature" odor. Aside from underscoring the rat's learning abilities and the apparent salience of the emissions resulting from the differential goal events relative to signature odors, this finding further suggests that the discriminative emissions are the same, or at least similar, among rats. Without the assumption of commonality, it would have to be concluded that the rats solved a very difficult multiple discrimination problem. That is, assuming idiosyncratic emissions, the cues would have changed in an unpredictable fashion from day to day, with sometimes different, sometimes the same, odor(s) appearing, and then sometimes singly and other times in pairs. The rat may be able to solve such problems, perhaps through a "learning set," but the commonality assumption seems more parsimonious. This lends support to the Eslinger and Ludvigson (1980b) conclusion.

When it is further noted that response patterning was more 
pronounced when rats occupied the third, as opposed to the second, squad position, the assumption of idiosyncratic emissions becomes even more cumbersome. In contrast, that is precisely what is expected under the commonality assumption, since the odor should be more concentrated following two rats, rather than one. However, it should be noted that the significance of the squad position data is not certain: if odors were disseminated farther down the runway for rats in the third position, then earlier detection might have produced slower speeds. Of course, both greater concentration and dissemination might have been factors.

The tendency for greater decrements in run speeds on $\mathbf{N}$ trials to accompany successively lower squad positions, suggesting an accumulation of $\mathbf{N}$ odor and greater response inhibition with greater amounts of $\mathbf{N}$ odor, implies that this odor is an important stimulus in this task. The absence of a comparable incremental effect on $\mathbf{R}$ trials may mean that $\mathbf{R}$ odors were not controlling responding, a response is less sensitive to variation in the concentration of an $\mathrm{S}+$ odorant than in that of an S- odorant, insignificant accumulation occurred, or a response ceiling prevented the effect from appearing. Taylor and Ludvigson (1980a) found that $R$ odor did control responding in a similar task, which argues against the first alternative but not the second. Prytula et al. (1981) found faster running to a buildup of reward odor from nine or more prior rats than from two or three, which suggests $\mathbf{R}$ odor accumulation; however, volatility may be such that accumulation from one to two rats is below threshold. In any case, the present data do not implicate an R odor.

Finally, it is worth noting that fully $70 \%$ of the individual variation in the magnitude of patterned responding when subjects occupied the third squad position was attributable to the number of opportunities to associate the odors with goal events. This demonstrates the strong effect of learning factors on the phenomenon.

\section{REFERENCES}

Capaldi, E. J. Memory and learning: A sequential viewpoint. In W. K. Honig \& P. H. R. James (Eds.), Animal memory. New York: Academic Press, 1971.

Eslinger, P. J., \& Ludvigson, H. W. Are there constraints on learned responses to odors from rewarded and nonrewarded rats? Animal Learning \& Behavior, 1980, 8, 452-456. (a)

Eslinger, P. J., \& Ludvigson, H. W. Commonality among rats in production of reward and nonreward odors. Bulletin of the Psychonomic Society, 1980, 16, 191-193. (b)

Ludvigson, H. W. Runway behavior of the rat as a function of intersubject reward contingencies and constancy of daily reward schedule. Psychonomic Science, 1969, 15, 41-43.

Ludvigson, H. W., \& Sytsma, D. The sweet smell of success: Apparent double alternation in the rat. Psychonomic Science, 1967, 9, 283-284.

McHose, J. H., \& Ludvigson, H. W. Differential conditioning with nondifferential reinforcement. Psychonomic Science, 1966, 6, 485-486.

Mellgren, R. L., Fouts, R. S., \& Martin, J. W. Approach and escape to conspecific odors of reward and nonreward. Animal Learning \& Behavior, 1973, 1, 129-132.

Morrison, R. R., \& Ludvigson, H. W. Discrimination by rats of conspecific odors of reward and nonreward. Science, 1970, 167, 904-905.

Prytula, R. E., \& Davis, S. F. Runway performance as a function of positively and negatively correlated olfactory cues. Psychological Reports, 1974, 35, 735-740.

Prytula, R. E., Davis, S. F., \& Fanning, J. J. The acquisition of a running response as a function of odor buildup, squad rotation, and introduction of naive subjects. Animal Learning \& Behavior, 1981, 9, 556-560.

Seago, J. D., Ludvigson, H. W., \& Remley, N. R. Effects of anosmia on apparent double alternation in the rat. Journal of Comparative and Physiological Psychology, 1970, 71, 435-442.

TAYLOR, R. D., \& Ludvigson, H. W. Selective removal of reward and nonreward odors to assess their control of patterned responding in rats. Bulletin of the Psychonomic Society, 1980, 16, 101-104. (a)

TAYLOR, R. D., \& Ludvigson, H. W. Selective removal of alleyway paper flooring or air to assess locus of nonreward odor. Bulletin of the Psychonomic Society, 1980, 16, 105-108. (b)

(Received for publication March 23, 1983.) 